\section{Buchrezension zu: Die Idee des Humanen}

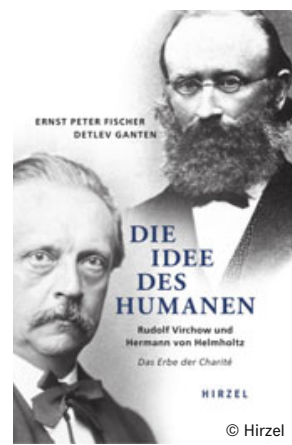

Die Idee des Humanen Rudolf Virchow und Hermann von Helmholtz

Das Erbe der Charité

Ernst Peter Fischer und

Detlev Ganten

263 S., Hirzel, 2021. HC, 22,90€

ISBN: 9783777629025

Auch als E-Book erhältlich

DOI: $10.1007 / \mathrm{s} 12268-021-1656-7$

(C) Der Autor 202

Der Titel des Buchs „Die Idee des Humanen“ ist „A Tall Order“, wie man in den USA so schön sagt, um sie auf gerade einmal 250 Seiten Text abzuhandeln. Ernst Fischer ist ein bekannter Wissenschaftshistoriker und Detlev Ganten ein bekannter klinischer Pharmakologe, der über viele Jahre das Max-DelbrückZentrum in Berlin leitete. Das Buch entstand zum Anlass des 200. Geburtstags der beiden groBen Forscherpersönlichkeiten Rudolf Virchow und Hermann von Helmholtz in diesem Jahr. Beide Forscher haben auf verschiedene Weise sehr wichtige Beiträge zur Medizin geleistet und haben unterschiedlich starke Wurzeln in Berlin.

Die große Stärke des Buchs liegt in den sehr lebendigen und einprägsam beschriebenen Biografien der beiden Protagonisten im Mittelteil auf den Seiten 73168. Dieser Abschnitt ist sehr lesenswert und zeigt gut auf, wie Virchow und Helmholtz, zwei ganz unterschiedliche Persönlichkeiten, ihren eigenen Weg gingen, um eine Wissenschaft mit Menschlichkeit voranzubringen. Diesen Teil kann man sehr gut unabhängig vom Rest des Buchs in wenigen Stunden lesen.

Warum betont der Rezensent dies? Im vorderen und hinteren Teil versuchen die beiden Autoren, „das Humane“ nicht nur im Zusammenhang mit Virchow und Helmholtz darzustellen, sondern im weiteren Sinne mit der Charité, der Wissenschaftsstadt Berlin vom 19. Jahrhundert bis zum heutigen Tage und darüber hinaus mit globalen Entwicklungen in der Wissenschaft bis hin zu CRISPR/ Cas (Emmanuelle Charpentier) sowie der SARS-CoV-2-Pandemie (Christian Drosten). Das ist es, was der Rezensent als „A Tall Order" bezeichnet. Es ist einfach sehr schwer, so viele unterschiedliche Aspekte der Wissenschaft auf dem naturgemäß begrenzten Platz eines typischen Sachbuchs unterzubringen. Daher bleibt das Buch im vorderen und hinteren Abschnitt oft recht oberflächlich und unfokussiert. Der Bogen ist sehr weit gespannt, aber „you can't get it all“ auf 250 Seiten.

Man merkt doch deutlich, dass das Buch von zwei sehr unterschiedlich denkenden Autoren geschrieben wurde. Wenn sich der Leser über die Biografien von Virchow und Helmholtz informieren möchte, so ist es eine kurzweilige und interessante Lektüre. Auch werden wichtige Aspekte der Wissenschaftsgeschichte und Wissenschaftsphilosophie angerissen, aber ohne ausreichend vertieft zu werden. Die erfahrenen Autoren sollten überlegen, ob sie die Thematik in weiteren fokussierten Büchern vertiefen. Der Preis des technisch sehr gut aufgemachten Buchs ist angemessen. Nützlich sind Zeittafeln zur Geschichte der Charité sowie ein Verzeichnis weiterführender Literatur.

Roland Seifert, Medizinische Hochschule Hannover, seifert.roland@mh-hannover.de Diese Rezension erscheint Open Access. *
Funding note: Open Access funding enabled and organized by Projekt DEAL. Open Access: Dieser Artikel wird unter der Creative Commons Namensnennung 4.0 International Lizenz veröffentlicht, welche die Nutzung, Vervielfältigung, Bearbeitung, Verbreitung und Wiedergabe in jeglichem Medium und Format erlaubt, sofern Sie den/die ursprünglichen Autor(en) und die Quelle ordnungsgemäß nennen, einen Link zur Creative Commons Lizenz beifügen und angeben, ob Änderungen vorgenommen wurden. Die in diesem Artikel enthaltenen Bilder und sonstiges Drittmaterial unterliegen ebenfalls der genannten Creative Commons Lizenz, sofern sich aus der Abbildungslegende nichts anderes ergibt. Sofern das betreffende Material nicht unter der genannten Creative Commons Lizenz steht und die betreffende Handlung nicht nach gesetzlichen Vorschriften erlaubt ist, ist für die oben aufgeführten Weiterverwendungen des Materials die Einwilligung des jeweiligen Rechteinhabers einzuholen. Weitere Details zur Lizenz entnehmen Sie bitte der Lizenzinformation auf

http://creativecommons.org/licenses/ by $/ 4.0 /$ deed.de. 